\title{
Research on Network Transmission and Exchange Technology of Digital Audio
}

\author{
Dapeng Gao \\ Beijing Wuzhou Shenyun Technology Co., Ltd., Beijing, China \\ Email address: \\ 13911150724@139.com
}

\section{To cite this article:}

Dapeng Gao. Research on Network Transmission and Exchange Technology of Digital Audio. International Journal of Information and Communication Sciences. Vol. 4, No. 4, 2019, pp. 68-74. doi: 10.11648/j.ijics.20190404.12

Received: September 22, 2019; Accepted: December 24, 2019; Published: December 26, 2019

\begin{abstract}
The application of modern science and technology in the dance beauty industry is more and more extensive. The application of network digital audio transmission technology in the sound reinforcement makes the control interface graphical, intelligent control, operation humanized, and improves the stability of the system. The application of network digital audio technology in the sound reinforcement system will be the development trend of modern audio technology. The popularization of digital audio network transmission system is imminent. This paper analyzes the application of network digital audio transmission technology, points out the significance of the application of network digital audio transmission technology in modern society, and then summarizes the advantages and disadvantages of different types of network digital audio transmission technology through comparative study of different application methods of network digital audio transmission technology, and then on the basis of the above research, the network The application protocol of networked digital audio transmission technology is explored. Through the practical research on DSP centralized and DSP distributed of network digital audio transmission system architecture, the paper summarizes the different types of network audio transmission protocol for different application occasions and their advantages and disadvantages, and puts forward effective suggestions for the application and promotion of networked digital audio transmission technology.
\end{abstract}

Keywords: Networking, Digital Audio Transmission, CobraNet ${ }^{\circledR}$, EtherSound ${ }^{\circledR}$

\section{Introduction}

With the development of science and technology, digital audio network transmission system is imminent. Digital audio application has been fully mature. Digital audio transmission system integrates the current application of program source playback, DSP processing, audio synchronous track storage, online broadcast, live broadcast and so on, which greatly improves the transmission efficiency [1]. In recent years, China's modern science and technology has entered a stage of rapid development, which has also promoted the progress of digital information technology, and has been widely used in all walks of life [2]. The application of networked digital audio transmission technology in sound reinforcement has the advantages of concise system line, simple construction and convenient maintenance; networked management and control are used in digital audio transmission, and the control interface is graphical and easy to operate; intelligent control can be realized, the operation is humanized, the workload of staff can be reduced, and the misoperation of personnel can be reduced; automatic backup of malfunction equipment can improve the use of the system [3]. Stability. The application of networked digital audio technology in sound reinforcement system will be the development trend of modern audio technology. On the basis of previous studies, this paper mainly discusses the choice of network-based digital audio transmission scheme, the problems in the transmission process, the solutions and the development direction.

\section{Significance of the Application of Networked Digital Audio Transmission Technology}

Today, in the 21 st century, the earth has been described as a global village, and large-scale building complexes are also 
common. Individualized buildings and intelligent buildings are emerging in an endless stream. With the passage of time, the rapid development of information science and technology, especially the popularization and development of computer technology and network technology in communications and transportation industry, has put forward higher requirements for the traditional analog sound reinforcement system [4]. In today's large-scale building complexes, the traditional analog sound reinforcement system can no longer cope with the complicated real-time audio broadcasting, recording, storage, simultaneous live media broadcasting and on-demand work. For large or intelligent building complexes, the distance between one building and another is one or two kilometers, several kilometers or even dozens to tens of kilometers. In the routing arrangement, the traditional way is to install the equipment in the main control room, but sometimes the distance between the main control room and the loudspeaker will be several kilometers or more.

\subsection{Problems and Shortcomings of Traditional Analog Sound Reinforcement System}

Wire laying requires long loudspeaker cables.

\subsubsection{Technically}

Technically, if the loudspeaker line is too long, it will consume a large part of the power. In order to solve this problem, it is necessary to adopt loudspeaker cables with thicker diameter and higher purity to reduce the line resistance, reduce power consumption, or increase the power of the power amplifier.

\subsubsection{Economically}

Economically, the wires in the analog sound reinforcement system originally accounted for a large part of the cost of the whole system, but now the loudspeaker line is longer, the diameter is thicker, the purity is higher, and the cost will rise substantially.

\subsubsection{In Design}

In design, technicians may separate the control room from the power amplifier room and place the power amplifier room near the loudspeaker, which can really save most of the expensive speaker cables. But new problems have arisen. The signal from the main control room to the power amplifier room is usually weak and low-level signal. In long-distance transmission, it is easy to be disturbed by lightning, electromagnetic wave and power system, thus resulting in the system noise being difficult to suppress [5].

\subsection{Advantages of Traditional Analog Sound Reinforcement System}

As for the maintenance of equipment, the equipment of traditional analog sound reinforcement system is basically installed in the same equipment room, and some system equipment is installed in the control room, so the staff can easily find out whether the equipment is malfunctioning, which has certain convenience and intuition for the maintenance of equipment.

\subsection{Shortcomings of Traditional Analog Sound Reinforcement System}

In the sound reinforcement network system of a large building complex, if the analog sound reinforcement system is used for design, there will be more than ten or dozens of equipment room. And the distance between equipment rooms is far. Every day, when the staff patrol, it is difficult to ensure timely feedback from the failure of equipment to the detection of equipment failure, and the probability of problems is greatly increased, which has caused a very serious problem to our technicians [6].

Today, in the 21 st century, the application of digital audio network transmission system is imminent. Digital audio application has been fully mature. The digital audio transmission system integrates the current application of program source playback, DSP processing, audio synchronous track storage, and online broadcast, live broadcast and so on. Digital audio transmission is also the direction of development.

This paper mainly discusses the choice of networked digital audio transmission scheme, the problems in the transmission process, the solutions and the direction of development.

\section{Application Mode of Networked Digital Audio Transmission Technology}

Generally speaking, there are several modes of networked digital audio transmission technology.

\subsection{Broadcast}

It is referred to as digital broadcasting. It uses digital encoding to transmit the traditional audio signal source, information from the live broadcasting room, or pre-recorded program signals. It uses network transmission or digital satellite microwave carrier to carry out regional coverage transmission. It needs to be equipped with mobile receiving equipment to modulate frequency, short wave and long wave broadcasting [7]. With the maturity of digitalization and networking, the mode of network broadcasting is becoming more and more mature. The APPs combined with platform interactive experience, mobile terminal, mobile phone terminal and PC terminal are also emerging in a variety of ways. Its basic structure is interactive communication between radio DJ, studio and audience through microwave, reflecting real-time and interactive platform.

This mode is only one-way transmission, or the client can only use smaller data streams for on-demand, so it is not within the scope of the discussion of networked digital audio transmission in this paper. 


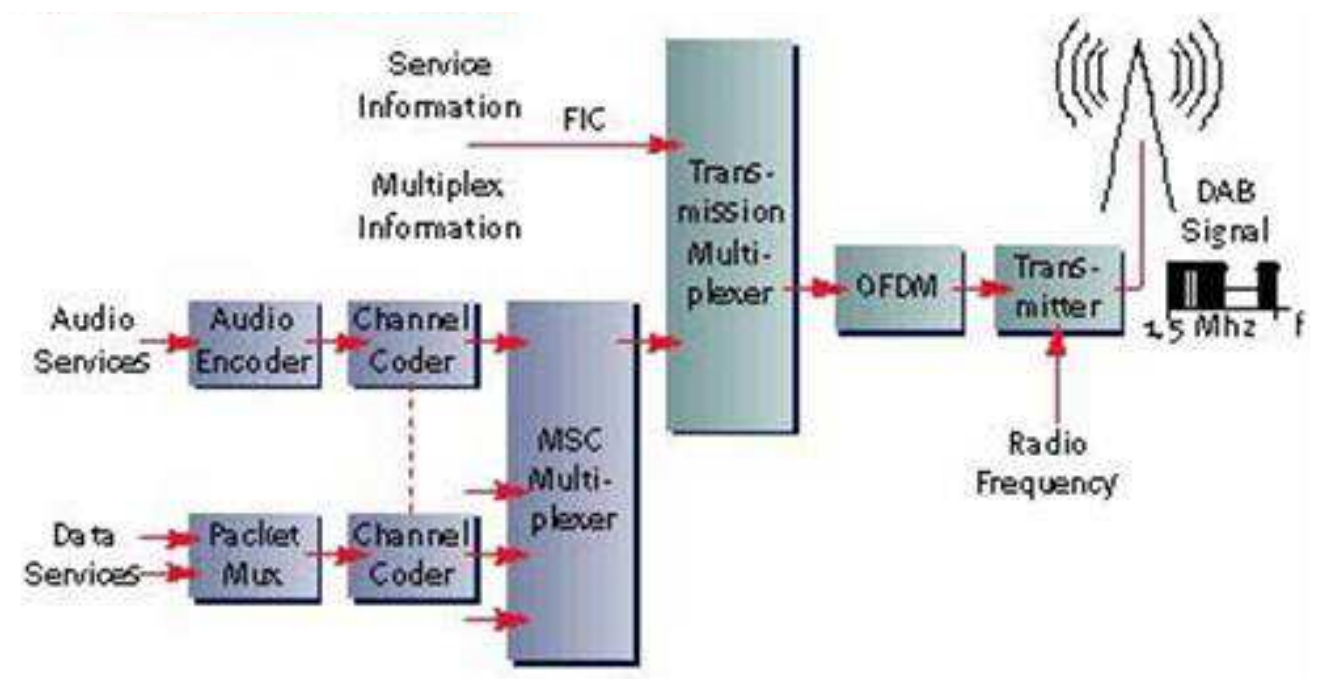

Figure 2: DAB Receiver

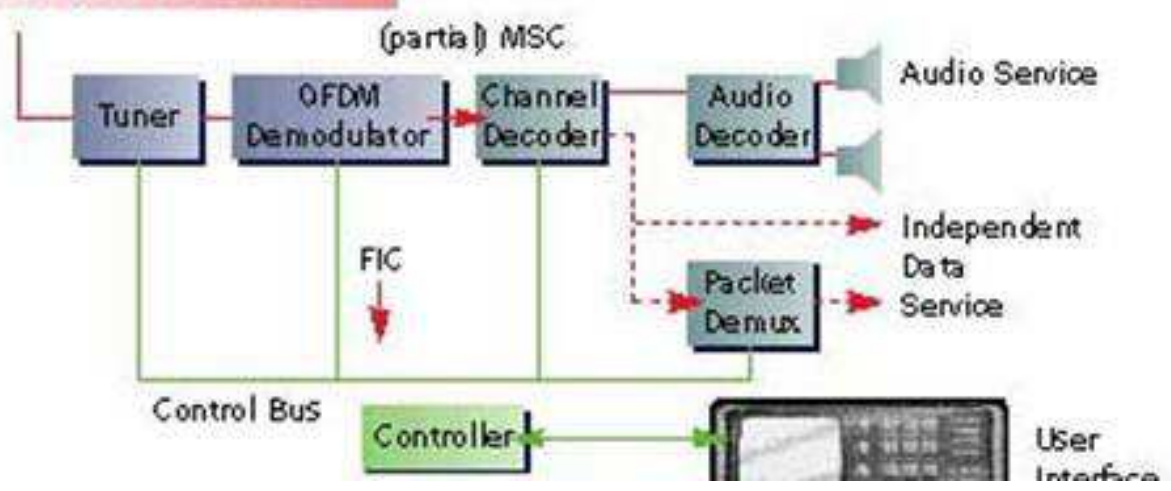

Figure 1. Broadcast mode.

\subsection{Point-to-point}

This mode has more use of transmission media, such as: SPDIF (optical fiber or coaxial), AES/EBU (shielded twisted pair), and DAAT (optical fiber). It uses point-to-point transmission, commonly known as unicast; or point-to-multipoint (distributed) transmission, commonly known as multicast.

Advantage: The digital delay is small and real-time is very good. Because of the point-to-point transmission, the bandwidth requirement of the transmission medium is relatively low, so the sound quality is better.

Shortcoming: Audio transmission is one-way, can only send audio data from one point to another point.

The above two modes are all networked digital audio transmission technology. However, because of the limitations of transmission mode, they cannot form an audio network, so they cannot be called networked digital audio transmission.

\subsection{Networked Digital Audio Transmission}

The networked digital audio transmission is a brand-new audio transmission mode with strong flexibility and expansibility.

What is network? The network is the latest medium and carrier in the information age. Whether in any corner of the world, as long as there is a network, it is as simple as standing opposite to each other. Through copper cables or optical cables, or even wireless satellites, a star-covered network is formed, which can be integrated in all directions to improve our work efficiency, life and entertainment, and information interconnection [8]. In terms of composition structure, it uses proprietary equipment to form a network, and then composes all levels of network to overlay. It can be understood that the whole earth is a large network, each country has its own network, each province and city has its own independent network, and each family has its own network.

So what is networked digital audio system transmission?

First of all, it is based on the network for transmission, which is the carrier of the media level, and secondly it is digital. Everyone knows that the number represents coding, such as 0101001 in computer language and so on. Again, it is a system, which means that it is not a single device, or a terminal, but a complex system composed of $\mathrm{AD}$ acquisition terminal, coding terminal, transmitting terminal, broadcasting terminal, DSP processing terminal, routing terminal Switch, receiving terminal, DA restore terminal, etc [9].

In the digital audio network, the network is like our street, 
which connects the information of households through copper or optical cables, and carries out accurate information transmission according to different door numbers and streets. All the useful information and conditional information enter the post office of the same region, and then a database system is formed. When the database system establishes various levels of authority, information sharing and resource sharing information are formed [10]. Digital audio network is a system that connects multiple audio devices with different geographic locations and independent functions through communication devices and lines, and realizes digital audio resource sharing with well-functioned network software (network protocol, information exchange mode, network operating system, etc.). Digital audio network is a complex system composed of $\mathrm{AD}$ acquisition terminal, coding terminal, transmitting terminal, broadcasting terminal, DSP processing terminal, routing terminal Switch, receiving terminal, DA restore terminal, etc. It allows all other devices to transmit data packets synchronously, queued and timely through sub-clock correction and time code synchronization. The data packet transmission mode can be either unicast of point-to-point or multicast of point-to- multipoint.

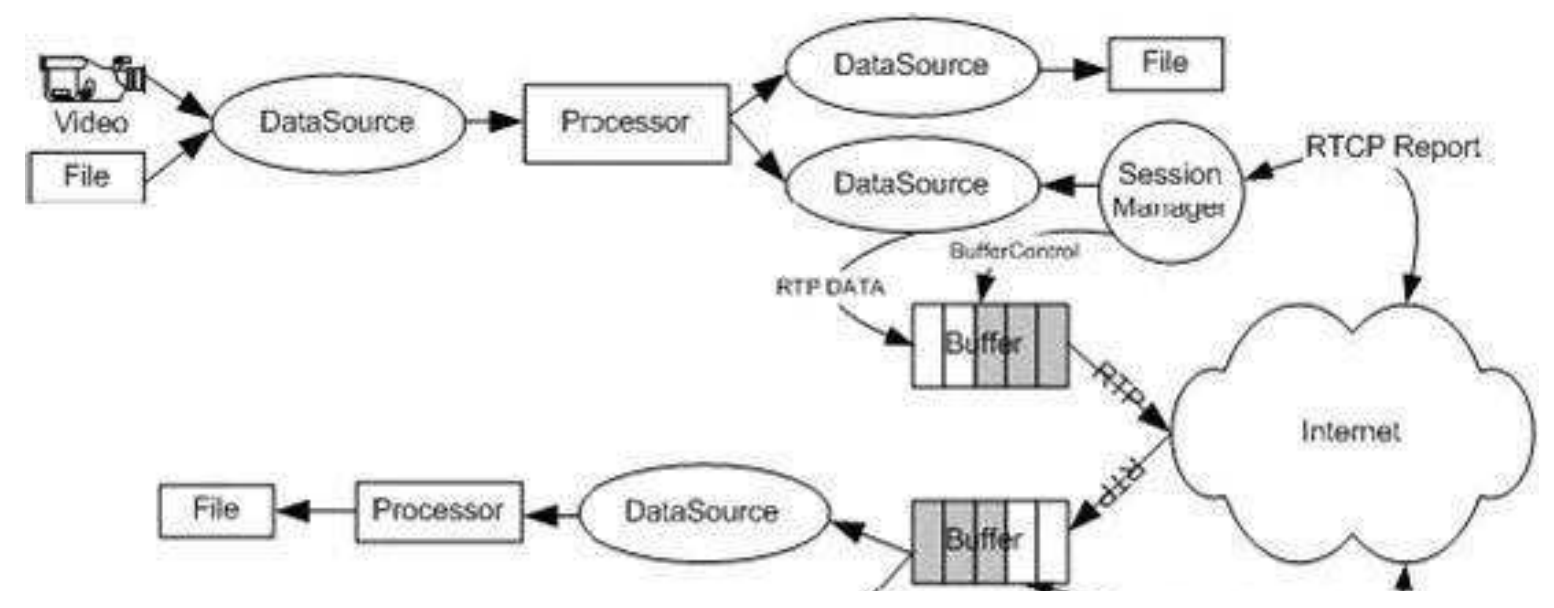

Figure 2. Network-style digital audio transmission.

It uses the advantages of network communication to collect media data and audio and video information, control information, power supply information and network data information to form a data stream, which is also called IP stream. It sets up a media server to store more media streams, and caches them on personal terminals for voice, video and data model reproduction [11]. Therefore, streaming media services are only used in the broadcasting of audio and video streams on the Internet, but cannot be used in the application environment with high real-time requirements for live performance or broadcasting.

\section{Classification and Characteristics of Networked Digital Audio Transmission Technology}

Networked digital audio transmission technology mainly relies on digital audio network. At the same time, network types are flexibly arranged and combined according to different requirements, which truly reflects the flexibility and openness of networked digital audio transmission technology.

Digital audio networks are classified into two types according to communication protocols:

Communication protocol based on Ethernet and Communication protocol based on privately-owned of each manufacturer.

Digital audio networks are classified into four types according to their topological structure: Bus topology; Ring topology; Star topology; Mesh topology.

The characteristics of digital audio network:

i. Reliability;

ii. Stability;

iii. Low delay rate;

iv. High sampling without distortion;

v. Backup and redundancy;

vi. Openness;

vii. Compatibility;

viii.Flexibility and expansibility;

ix. Highly integrated processing capability;

$\mathrm{x}$. Energy conservation and environmental protection.

Advantages of digital audio network:

It not only solves the problem of digital transmission of audio, but also provides high integration capability. It develops different types of digital audio network products for various manufacturers of digital network equipment and applications and leads the leading position and future development direction of the industry. It is no longer the accumulation of equipment the ocean of cables. A small network line solves all the applications of audio system, which reflects the charm of information technology from the front-end digital acquisition, coding, transmission, decoding and restoring [12]. It is well prepared for the full integration of IT, and its continuing openness also provides unlimited possibilities for our respective application requirements. It enhances the user experience and strides a historic leap forward to the future audio development. It has led the scientific exploration of human to the loudly, real and beautiful audio system. 
As for the core products of digital audio network system, each manufacturer and giant is more proficient in producing and developing digital audio processor equipment, which sets the whole process of digital audio signal acquisition, sampling, analog-to-digital conversion, DSP processing, digital-to-analog restoration, etc.

Digital audio processor integrates many functions such as audio matrix, automix and control. Its signal processing includes compress/limit, EQ, delay and other functions [13]. It has the characteristics of integration, intelligence, portability, maintainability, etc. It has become the mainstream equipment and core processing equipment in the audio industry.

\section{Application Protocol of Networked Digital Audio Transmission Technology}

\subsection{Network Transport Protocol}

TCP: A connection-oriented transmission mode with slow transmission speed but reliable. It has a mechanism of retransmitting for interrupts and packet dropouts.

UDP: A non-connection-oriented transmission mode with fast transmission speed but unreliable. It has no retransmitting for packet dropouts and error correction, so it is mostly used for broadcasting data packets.

The network transport protocol is shown in figure 3 .
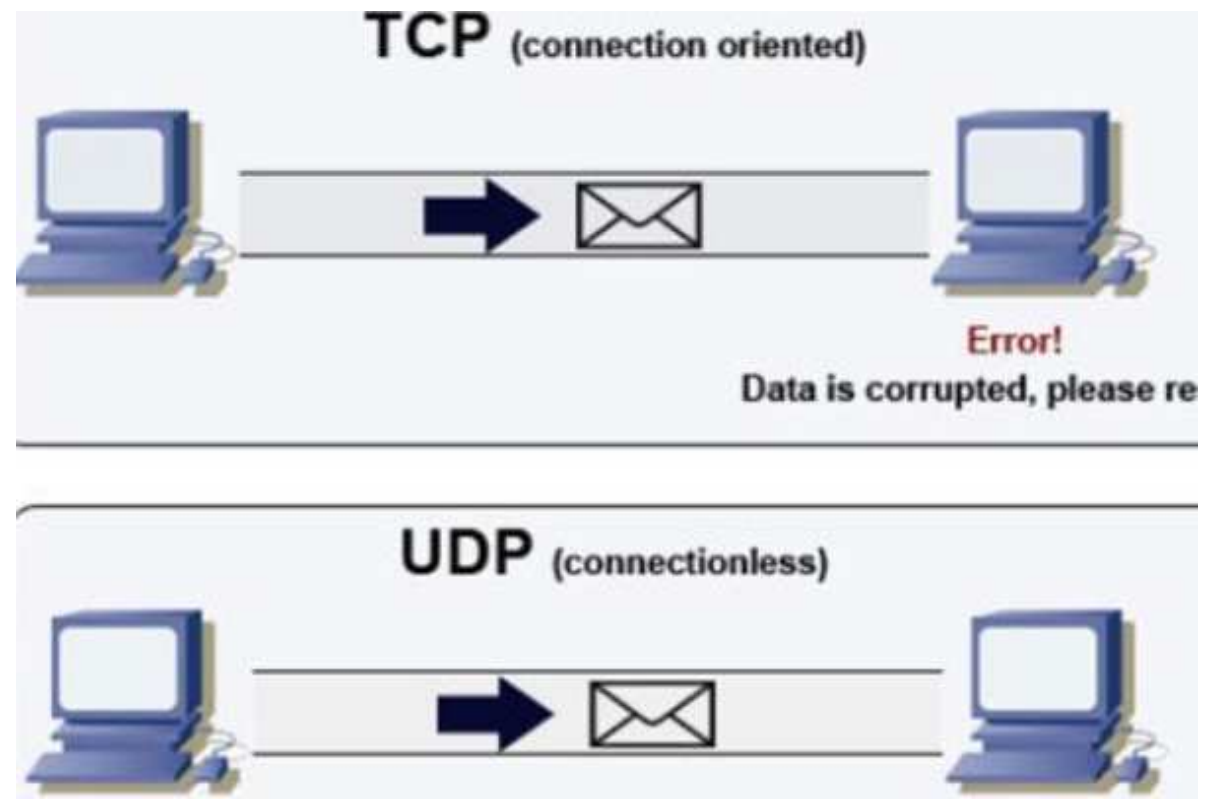

Figure 3. Network transport protocol.

\subsection{Audio Dedicated Transmission Protocol}

\section{CobraNet $\mathbb{R}$}

It was applied in the industry many years ago and became the industry standard.

The technology was developed by Peak Audio and authorized by Cirrus Logic to use by various audio manufacturers to develop various hardware products.

In view of the technical barriers in this protocol and the lack of further development in the later stage, the product equipment gradually withdraws from the audio market stage. It provides a strong cornerstone for the development of the industry.

EtherSound ${ }^{\circledR}$

The product follows the characteristics of ultra-low delay, and is used in various performance and entertainment venues, and is popular for a while.

It must be operated in an independent private network, and the extended functions are limited to a certain extent.

At the same time, "s" or snake-shaped network is used to transmit audio signals.

It uses general network equipment to deploy, and has been praised by many manufacturers.

For the one-way transmission problem in networking, private protocols do not really realize the function of network transmission, which needs further improvement and development.

Dante ${ }^{\circledR}$

It is the most stable protocol system current used to replace CobraNet, including audio information, control information, etc.

It supports gigabit network and TCP/IP routing, and can support different sampling frequencies and audio data streams in the same level network.

It is easy to use, install and maintain.

It has compatibility problems for different brand devices.

Q-LAN ${ }^{\circ}$

It is a private protocol, exclusive to QSC.

It is based on the third layer of network development and application.

It has high speed and low delay.

It has a friendly interface to develop and open data packets. 
It has compatibility problems for different brand devices. AVB $\AA$

It is an industry collaborative open standard protocol product.

It synchronously opens audio, video, control, data and power transmission.

It has ultra-high speed and ultra-low delay.

\subsection{Comprehensive Analysis}

The earliest CobraNet produced in 1996 has gradually faded out of the market. For the one-way transmission of EtherSound in star networking, there are new transmission modes recently, among which Dante, Q-lan and AVB are more mature and widely used.

Q-lan is a pure digital audio network developed by QSC Company in the United States, which is based on gigabit network processing technology. It consists of core processor and interface message processor. It is connected with a gigabit network in the middle. Its network delay is 0.667 milliseconds (5.33 milliseconds of CobraNet), maximum network throughput is 10 hops ( 7 hops of CobraNet), maximum input and output capacity is $512 \times 512$ channels $(32 \times 32$ channels of CobraNet). Q-lan considers the security and hot backup of the system at the beginning of the system design. Each device of Q-lan has two network interfaces, which can backup each other. There is no master-slave division between the two networks. When one network fails, it is switched to another network for use. Even if the original network is restored, it is no longer automatically switched back, which can avoid the unstable problem caused by repeated switching in the case of bad network contact [14].

AVB is a technology developed jointly by many professional audio and video manufacturers in the United States. Its characteristic is that in the transmission of network, it is not only the real-time transmission of audio, but also the packaging and transmission of video signals. AVB follows the standard of IEEE 802, ensures bandwidth, ultra-low latency and time synchronization on the basis of LAN, private network and WAN, provides perfect solution and strategy algorithm, and supports the information transmission of audio, video, control, power supply and data. It is the frontier and direction of the future development of the industry to ensure the timely transmission of network information while ensuring the simultaneous transmission of audio and video signals.

In addition, AVB devices need to use the core switching devices of special technology for transmission. They all have their own independent transmission protocols, but they do not use the conventional TCP/IP network protocol for transmission. Therefore, this kind of signal cannot be transmitted only through dedicated signal transmission equipment other than general switches [15]. It is relatively expensive, which is the main factor restricting its development. We believe that this technical problem will be solved in the near future.

\section{Distribution of DSP Resources for Networked Digital Audio Transmission}

In terms of system architecture, there are two common modes: centralized DSP and distributed DSP.

The centralized DSP is applied by Budweiser, BIAMP, QSC and other manufacturers. The audio processing in the whole network is completed on the core processor. The nodes of each network only do DA/AD audio conversion, and encode and transmit according to the relevant network protocol. When the system's DSP resources are insufficient, it is necessary to increase the core processing host, and distribute the resources equally in the system. The overall cost is relatively high.

The distributed DSP is applied by YAMAHA, Simei and other manufacturers. Every device in the network is equal. They have different number of DSP resources according to the number of interfaces. The advantage of this mode is that when the system adds more input and output interfaces, the number of DSP resources will increase correspondingly, and there will be no lack of DSP resources that cannot be allocated.

So when we make design choices, we try to choose the system architecture of distributed DSP.

The networked digital audio signal transmission is not only the transmission of digital audio signal, but also the transmission of control signals using the remaining bandwidth, such as RS232, RS422, RS485 signals, which can be packaged in the digital audio network for transmission, making a network not only for the transmission of digital audio signal, but also for the transmission of control signal.

\section{Conclusion}

Through the above research and analysis, network digital audio signal transmission, not only the transmission of digital audio signal, but also the transmission of control signal. In order to make the audio transmission more convenient and fast, it is necessary to not only do the transmission of digital audio signal in a network, but also do the transmission of control signal. Using DSP distributed system architecture, through digital audio network, to control other back-end equipment such as power amplifier or other stage equipment, lighting equipment, etc. can achieve these functions well. The network digital audio transmission technology is applied in the sound reinforcement, the system circuit is simple, the construction is simple, the maintenance is convenient; the network management and control, the control interface is graphical, the operation is convenient; the intelligent control, the operation is humanized, the workload of the staff is reduced, the misoperation of the staff is reduced; the fault equipment is automatically prepared, and the system use stability is improved. The application of network digital audio technology in sound reinforcement system will be the development trend of modern audio technology. 


\section{References}

[1] Yuanchang Wang. Advantages of digital audio and decoding technology in high fidelity transmission and radio and television engineering [J]. China New Communications, 2018, 20 (04): 140-141.

[2] Xiaoli Bai. Application of digital audio and its embedding technology in radio and television engineering [J]. Media Forum, 2019, 2 (06): $121+124$.

[3] Zhang Zhe. Digital Audio Signal Transmission Technology [J]. Communication World, 2017 (09): 106-107.

[4] Wang Kun. Application of Digital Audio Matrix Technology in Program Transmission [J]. Wireless Interconnection Technology, 2017 (08): 5-6.

[5] Hongshen Zhao, Xiankai Meng. Exploration on the construction of program source transmission system of transmitter by using network digital audio transmission technology [J]. Western Radio and Television, 2017 (04): 234.

[6] Jie Wang, Chengcheng Yang, Guangquan Yang, Hongrong Chen. Design of Digital Audio Transmission System for Concert Hall of a Provincial Grand Theatre [J]. Journal of Guangzhou University (Natural Science Edition), 2016, 15 (04): $57-60+95$.

[7] Caiyan Lu. Design and Implementation of High Performance Digital Audio Transmission System [J]. Computer Programming Skills and Maintenance, 2016 (03): 78-79.
[8] Meng Yang, Wei Lu, Yuan Song. FM Broadcasting and Relay System for Transmission of Baseband Digital Audio Signals [J]. Electroacoustic Technology, 2015, 39 (07): 40-43+84.

[9] Minghui Zhou. Analysis and construction of radio broadcasting system based on audio transmission [J]. Electro-acoustic technology, 2015, 39 (06): 70-74.

[10] Dan Yu, Wen Sun. Development of Digital Audio Wireless Transmission System [J]. Science and Technology Perspective, 2015 (14): 202.

[11] Zhi Wu. Theater Digital Audio System Transmission Network and Its Application [J]. Architectural Electricity, 2010, 29 (07): 60-64.

[12] Guicheng Liao, Jianhua Wei. Design and implementation of high performance digital audio transmission system $[\mathrm{J}]$. China Cable Television, 2010 (02): 165-168.

[13] Zhiyuan $\mathrm{Xu}$. Network transmission and exchange of digital audio [J]. Southeast Communication, 2005 (10): 78-79.

[14] PeakAudio Company. CobraNet Technical White Paper [M]. PeakAudio Company, 2012.

[15] Dong He, Sheng Huang, Charles Wang. Next Generation Networked Real-time Audio and Video Transmission Technology-Ethernet AVB [M]. Beijing: Publisher is unknown, 2017. 